\title{
Modelling the Fringing of the FORS2 CCD
}

\author{
J. R. Walsh ${ }^{1}$, H. Kuntschner ${ }^{1}$, E. Jehin ${ }^{2}$, A. Kaufer ${ }^{2}$, K. O'Brien ${ }^{2}$, \\ M. Riquelme ${ }^{2}$, and A. Smette ${ }^{2}$ \\ 1 ESA, Space Telescope European Co-ordinating Facility, \\ Karl-Schwarzschild-Strasse 2, 85748 Garching, Germany; jwalsh@eso.org, \\ 2 ESO, Alonso de Cordóva 3107, Vitacura, Santiago, Chile
}

\begin{abstract}
Thinned CCD detectors display fringing which arises from the interference of multiply reflected light in the layers of the CCD. If the layer construction - the thicknesses and refractive indexes of the layers - is known, then the observed fringing can be accurately modelled and used to correct imaging and spectroscopic data for its effects. In practice the specifications on the actual deposited layer thicknesses may not be known to sufficient accuracy to predict the fringe behaviour. Thus calibration data, in the form of monochromatic flat fields, is required and can be modelled using the technique outlined by Malamuth et al. 2003, which has been applied to ACS CCDs.

Initial tests to model the observed fringing of the FORS2 MIT CCD are described. A set of six monochromatic flat fields was generated by shining light from a tunable monochromator into the FORS2 instrument calibration unit. Peak-to-peak fringe amplitude of 7-8\% was measured at a wavelength of $970 \mathrm{~nm}$. In order to model the CCD structure, a series of monochromatic flats at closely spaced wavelengths is required, which would have been very time-consuming to acquire with the monochromator. An alternative strategy is outlined. The fringing characteristics of CCDs should be systematically calibrated while still in the test laboratory.
\end{abstract}

\section{Observational Setup}

A commercial monochromator was used to feed monochromatic light into the FORS2 calibration unit using a fibre. The slit of the monochromator was set to $250 \mu \mathrm{m}$, which produced a beam of width slightly less than $1.0 \mathrm{~nm}$. Given the wavelength period of the fringing of around $3 \mathrm{~nm}$, this provides satisfactory sampling of the fringes. Table 1 provides brief details of the observations.

In order to determine the actual wavelength of the monochromatic light illuminating the detector, each monochromatic image was accompanied by an exposure with the $600 \mathrm{z}+23$ grism, with OG590 order sorting filter, and a 0.3 arcsec slit. Wavelengths were simply determined by linear interpolation, using a linear dispersion calculated from the positions of the 885.387 and $865.438 \mathrm{~nm}$ lines on an arc lamp exposure (dispersion determined as $0.0803 \mathrm{~nm} / \mathrm{pix})$. 
Table 1. Observational details

\begin{tabular}{llll}
\hline $\begin{array}{l}\text { Meas. } \\
\lambda(\mathrm{nm})\end{array}$ & $\begin{array}{l}\text { Meas. } \\
\text { FWHM }(\mathrm{nm})\end{array}$ & $\begin{array}{l}\text { Exp. } \\
\text { Time }(\mathrm{s})\end{array}$ & $\begin{array}{l}\text { Fringe } \\
\text { Pk-to-Pk }(\%)\end{array}$ \\
\hline 774.0 & 0.84 & 60 & \\
876.0 & 0.75 & 1800 & 2.2 \\
906.0 & 0.65 & 1800 & 3.0 \\
926.1 & 0.60 & 1800 & 5.1 \\
956.1 & 0.75 & 1800 & 7.0 \\
986.0 & 0.75 & 1800 & 7.5 \\
\hline
\end{tabular}

\section{Reduction}

The monochromatic flats were simply reduced by subtracting a constant value of 185 for the bias value and normalised by dividing by a 2D Gaussian smoothed ( $\sigma=30 \mathrm{pix}$ ) version of the bias-subtracted image. The smoothing $\sigma$ was determined by examining the rms of a selected region of the chip. Too low values of $\sigma$ fit the fringe scale (around 40 pixels peak-to-peak for the closest spaced fringes), while large values of $\sigma$ would not remove the large-scale illumination pattern, which was peaked to the lower right of Chip 2 (slave). Figure 1 shows four images, normalised to unity by the smoothed version and hereafter referred to as fringe flats. The flat at $774 \mathrm{~nm}$ had low signal-to-noise and no fringes were visible, so it is not shown.

\section{Results}

An indication of the peak-to-peak amplitude of the fringing is listed in Table 1 , measured in a region where the fringing is highest (around pixel 1300,1300 on the master chip). Whilst the fringing increases considerably from 900 to $960 \mathrm{~nm}$, the increase levels off to higher wavelength. Figure 2 shows the fringe contrast in two locations on the master and slave chips, again chosen to have high fringe amplitude. The crosses in Fig. 2 derive from the positions selected to have a high value in the $956 \mathrm{~nm}$ fringe map, whilst the triangles are from a nearby position (in the adjacent valley on the $956.0 \mathrm{~nm}$ image).

Shown in Fig. 2 (right) is a possible set of fringes. These have been derived by taking the wavelength period of the fringing to be $2.9 \mathrm{~nm}$, as measured from some 300I flats taken in the ESO GOODS programme, and approximating the fringing by a simple sine function. An envelope of the fringe amplitude with wavelength, which would be produced by a thinner layer deposited on the Silicon, is approximated by a $\sin ^{2}$ function. This is purely illustrative; there is not sufficient data at closely spaced wavelengths to determine if the period of the fringing varies across the detectors or if the envelope of the fringe amplitude is more complex (see for example Malamuth [1] for STIS and Walsh et al. [2] for ACS detectors). 

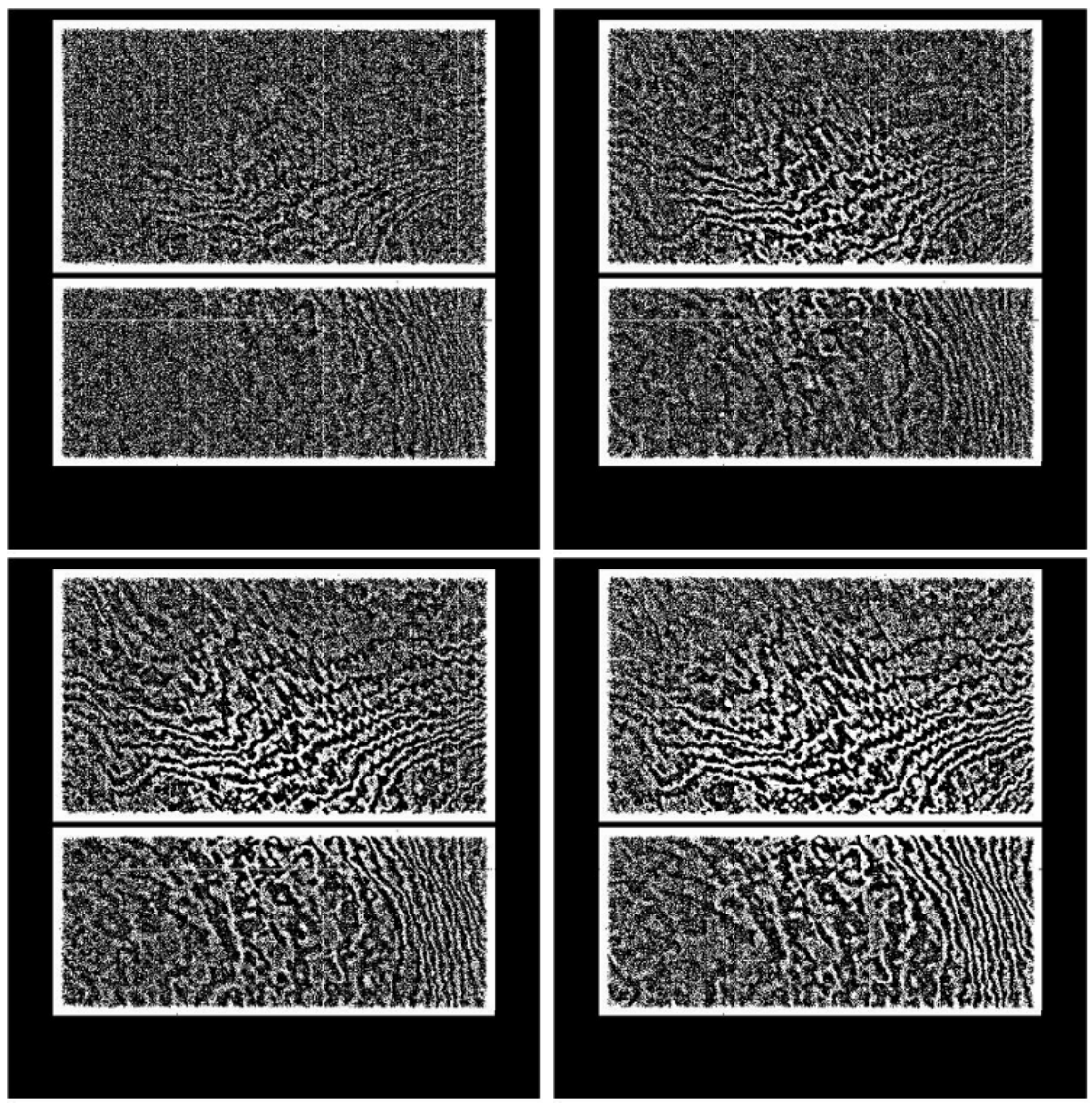

Fig. 1. Set of FORS2 fringe flats at wavelengths of 876, 906, 926, $956 \mathrm{~nm}$
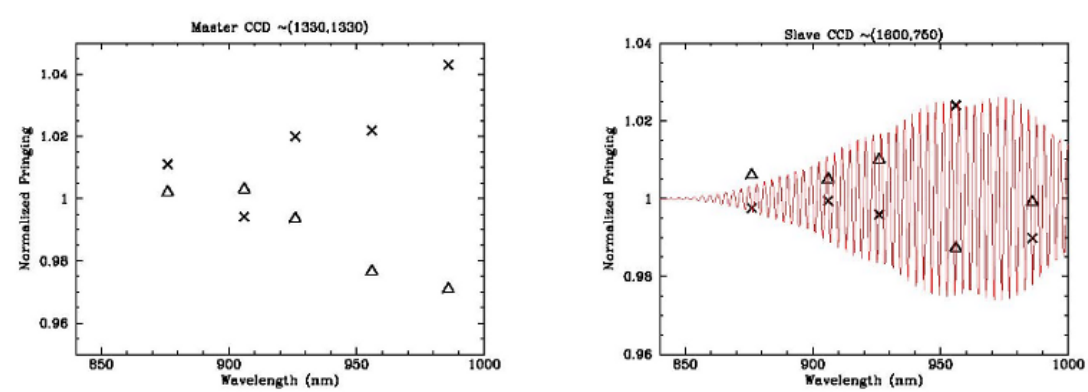

Fig. 2. Fringe contrast at two positions on the FORS master chip around pixel $(1330,133)$ are shown (left). Fringe contrast at two positions on the slave chip around pixel $(1600,1700)$ are shown $($ right $)$. A possible set of fringes is shown for illustration (not a model) 


\section{Prospects}

The fringe flats with the monochromator provide excellent data for modelling the fringing through fitting of the CCD layer thicknesses, given a knowledge of CCD composition and the refractive index of the layers (Malamuth et al. [1]; Walsh et al. [2]). Walsh et al. [2] modelled the layer structure of the HST Advanced Camera for Surveys (ACS) High Resolution Channel (HRC) CCD, manufactured by SiTe, using multiple monochromatic full field flats. In order to perform such modelling, it is necessary to have a closely-spaced sampling of wavelengths. For the case of the FORS2 CCD then in order to sample the fringe period adequately, monochromatic sampling about every $0.7 \mathrm{~nm}$ would be required, at least at a moderate number of wavelengths. This closely-spaced sampling can be supplemented by fringe flats at other isolated wavelengths in order to determine the envelope of the fringe amplitude. Given the typical exposures of $30 \mathrm{~min}$ which were required to reach counts of 5000 15000 electrons per unbinned pixel (lower to redder wavelengths) with the monochromator and FORS calibration unit, then another approach needs to be considered for detectors mounted on operational instruments.

The suggested approach would be to use a grism and the MOS single long slit mode spanning all the multi-slits, placing the slit at many positions over the detector to provide flat field illumination at many wavelengths for each pixel. The $600 \mathrm{z}$ grism would be well suited, or the 300I. The dome lamp could be used and a high signal could be quickly reached, allowing dense sampling of wavelengths over a few regions and sparser sampling of other wavelength regions for each pixel, in a relatively short time. With a choice of grisms, coverage of the wavelength region with highest fringing amplitude (950-1000 nm) could be achieved. A concern here is that the rail between the slitlets obscures some pixels. The obscuration between slitlets is at maximum $3(2 \times 2$ binned $)$ pixels. In the worst case no useful fringe information is available across these regions. When modelled, the detector layer thickness could be interpolated across these regions; probably the CCD layer thicknesses vary fairly smoothly, so the loss in fidelity may not be important.

A few full-field deep monochromatic flats could be taken in addition to the scanned slit data as a minimum check. There must be some concern that the different beam, entering the telescope from dome flats as compared to the astronomical objects (and sky), may alter the measured fringing behaviour. Some on-sky data should also be analysed. An out of focus image of a bright star could be trailed along the MOS slit at a number of slit positions as a check. Similar procedures for determining the fringing of the VIMOS CCD's could be considered using aligned slitlets or specially designed masks.

Ideally the set-ups to measure fringing could be performed in the CCD test laboratory away from the limitations of an operational observatory environment. Important here would be to simulate the input beam on the detector appropriate for the particular spectrometer. 


\section{References}

1. E.M. Malamuth, R.S. Hill, T. Gull, et al.: Publ. Astron. Soc. Pacific 115, 218 (2003)

2. J.R. Walsh, W. Freudling, N. Pirzkal, A. Pasquali: Modelling the fringing of the ACS WFC and HRC chips. ACS ISR 03-12 (2003) 